\title{
Experimental evaluation of needle tip force sensing associated to tactile feedback for improving needle remote insertion
}

\author{
C. Saudrais ${ }^{1}$, L. Rubbert ${ }^{1}$, L. Bonnefoy ${ }^{2}$, R. Zhu ${ }^{3}$, H. Schneegans ${ }^{2}$, C. Baur ${ }^{2}$, \\ U. Mescheder ${ }^{3}$, P. Renaud ${ }^{1}$ \\ 1 AVR-ICube, Strasbourg University, INSA Strasbourg, France \\ pierre.renaud@insa-strasbourg.fr \\ http://spirits.icube.unistra.fr/en/ \\ 2 Instant-Lab, EPFL, Switzerland \\ 3 Hochschule Furtwangen, Germany
}

\begin{abstract}
In this paper, we present an experimental evaluation of tactile feedback for remote needle insertion in soft tissues. A needle with tip force sensing is used to detect changes of tissues and puncture events during insertion. This information is provided to the user during teleoperation by means of a tactile display. Evaluation is conducted by reproducing needle insertion as performed during biopsy procedures in the liver. Needle tip force sensing makes the measurement of tissue change and puncture easier compared to force measurement at the base of the needle. Puncture events are correctly understood by the users. Change of tissue is also satisfactorily detected, which could be an interesting additional information for the user to perform surgical gesture.
\end{abstract}

Keywords: interventional radiology $\cdot$ needle insertion $\cdot$ tactile feedback $\cdot$ force sensing

\section{Introduction}

In interventional radiology, the use of X-ray based imaging devices represents a safety issue for radiologists due to radiations. MRI scanners also have the issue of limited access to the patient due to the bore geometry. Remote manipulation of needle could then improve the conditions for tasks such as biopsy performed by radiologists with these modalities. Radiologists achieve needle insertion relying on images and also insertion forces [4]. Because of the multiple visual sources of information in the operating room that the radiologist has to deal with, remote manipulation with force feedback to inform about the puncture of tissues and the change of tissues are then of interest [1]. In the following we focus on this objective, considering kidney and liver biopsy procedures. Such procedures consist in local anesthesia at the biopsy needle insertion point, before skin incision. A coaxial biopsy needle is then inserted after deep anesthesia along the planned path. The biopsy needle trajectory is monitored with a medical imaging device when going through abdominal muscle, fat and the organ of interest. When the needle tip reaches the point of interest, the biopsy is performed with a trigger mechanism before extracting the needle carrying the desired tissue specimen [2]. 
Teleoperation with force feedback to reflect insertion forces during the needle progression is possible, but challenging in terms of control [3]. An easier approach is to provide to the user only specific events such as the puncture of membranes surrounding tissues like muscle or liver, that also possess different stiffnesses and mechanical properties [4]. In [1], we showed that we were able to measure in lab conditions the punctures of membranes with great accuracy thanks to a Fabry-Perot load cell placed inside the needle, close to the tip. In [5], we investigated how to render information with a tactile feedback using a dynamic Braille display. In this paper, their combination is evaluated experimentally to investigate the capacity of users to understand change of tissues and occurrence of punctures during a needle insertion, and therefore to add to the medical image additional information with tactile feedback.

\section{Methods}

\subsection{Set-up}

A $16 \mathrm{G}$ needle (diameter of $1.6 \mathrm{~mm}$ ) with tip force sensing capability, which has been presented in [1] is inserted using a linear table (FB075, Nanomotion). To reproduce the biopsy, the needle insertion is performed at constant speed in a phantom composed of 3 layers (Fig. 1). These layers are produced with gelatin, using concentrations of 7\%, 45\% and $18 \%$ [6] to reproduce respectively the bio-mechanical properties, i.e. stiffness, of fat, muscle and organ (liver or kidney). The layers are separated by membranes composed of polyethylene cling film in order to reproduce the faciæ existing between the anatomical structures. The signal associated to the membrane puncture can be delivered to the user or not, so that three scenarios can be reproduced with two, one or no membrane between the layers. The phantom is rotated after 3 insertions that are shifted radially by $8 \mathrm{~mm}$, so the insertions are never performed in the same volume of the phantom. $22.5^{\circ}$ rotations are applied, therefore 48 insertions can be performed with a single phantom.

The Fabry-Perrot load cell [1] located in the instrumented needle is connected to a FISO $^{\text {тM }}$ white light interferometer. The signal is then transferred to a PC for signal processing using Labview platform. For some needle insertions, force measurement at the base of the needle is performed for comparison purpose using a force sensor Scaime K1107 mounted between the needle and the linear table. The tactile feedback is provided by means of a so-called tactuator, composed of a Braille display based on a tactile actuator SC5 (KGS Corporation). The tactile display is an array of $8 \times 8$ dots $\left(25 \times 25 \mathrm{~mm}^{2}\right)$ to produce dynamic patterns using an Arduino platform. A push button is also provided to the user to record puncture events. According to the initial results described in [5], the following two patterns are being used (Fig. 3): a moving horizontal line which frequency varies with the nature of the gel as detected with the needle tip, and a single ON/OFF signal generated at each puncture detection. The frequency varies proportionally with the measured force between $10 \mathrm{~Hz}$ and $25 \mathrm{~Hz}$. In order to set the frequency range, one insertion was used to determine the minimum value of force, associated to $10 \mathrm{~Hz}$, and the maximum value, associated to $25 \mathrm{~Hz}$. 


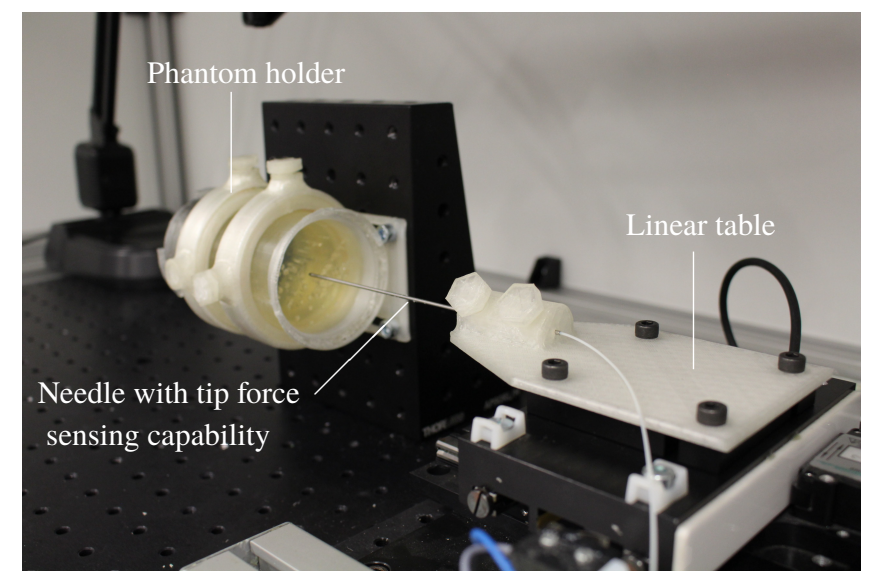

Fig. 1. The linear table holding the needle with tip force sensing capability and the phantom.

\subsection{Protocol}

The evaluation is performed on 11 healthy subjects ( 9 male and 2 female subjects) between 22 and 47 years old. Each subject is first introduced to the protocol and the tactile feedback using a prerecorded sequence, in order to get familiar with the dynamic patterns generated with the tactuator. The training phase is two and half minutes long, to keep the learning phase short. Each subject is equipped with noise-canceling headset (Sony WH-1000XM3) and is placed in such a manner he cannot see nor hear the needle progression. Insertion start is indicated to the subject by a visual sign. When the subject considers a membrane puncture occurred, he presses the push button. In addition, he mentions orally if a tissue change occurs. After each insertion, the subject leaves the room keeping the noise canceling headset while the next insertion settings are prepared.

In order to avoid learning of the sequence by the subject, the insertion sequences are generated randomly, modifying two parameters. First, the insertion speed is set to $2 \mathrm{~mm} / \mathrm{s}$ or $4 \mathrm{~mm} / \mathrm{s}(\mathrm{S}=1$ or $\mathrm{S}=2)$, so the time to go through a layer is not necessarily constant between two experiments. Second, the presence of the number of membrane (up to two) is not always reflected to the subject. Puncture of the first membrane (M1=0 or M1=1) and the second membrane $(\mathrm{M} 2=0$ or $\mathrm{M} 2=1)$ are varied between two experiments. A total of 8 combinations of conditions S, M1, M2 exists. For each subject, 3 insertions are achieved choosing randomly a set of conditions. The subject is only aware of the number of layers in the phantom, and the possible presence of membranes.

\section{Results}

\subsection{Needle tip force sensing performances}

In Fig. 4, the force measurements as functions of the needle progression are represented for insertions at speeds of $2 \mathrm{~mm} / \mathrm{s}(3$ insertions) and $4 \mathrm{~mm} / \mathrm{s}$ ( 3 insertions). The membrane 


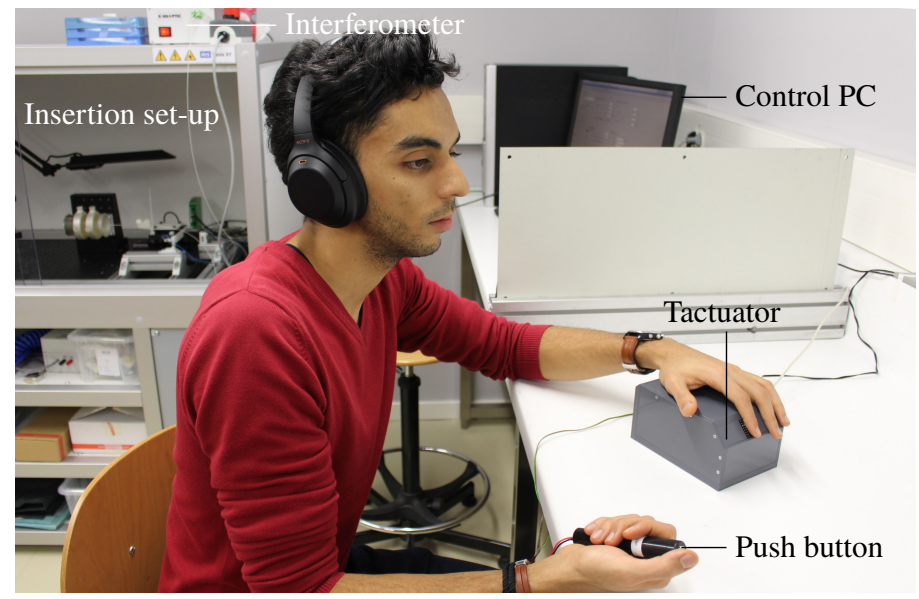

Fig. 2. Experimental set-up during evaluation with subject.

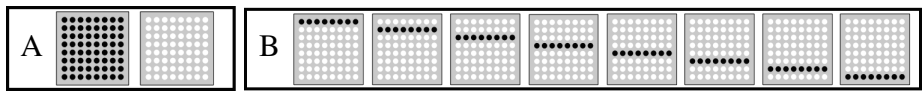

Fig. 3. Patterns for the puncture event (A) and the change of tissue (B).

punctures M1 and M2 are recorded synchronously for all measurements as expected. The evolution of the tip force is not dependent on the speed and needle insertion depth whereas the proximal force profiles exhibit different slopes. Using the tip force, it is quite simple to discriminate the nature of the layers. On the contrary the proximal force measurement is dependent on the needle velocity, so it is difficult then to discriminate the layers. In addition, after noise signal filtering, membrane puncture appears clearly on the tip force measurement, while for the first membrane it can be seen that the peak of proximal force measurement is hardly visible.

\subsection{Experimental evaluation}

The Table 1 provides the results of the experimental evaluation with the tip force sensing needle (distal). The experiment has been performed on two different days with thus two different phantoms, but with the same gel compositions. The first 7 subjects starting from the left column of the table did the experiment the first day in a row, and the 4 remaining on the second day in a row.

As a summary, 65 out of 66 membrane puncture detections (98\%) have been successfully performed. 59 out of the 66 frequency changes (89\%) have been successfully detected. 


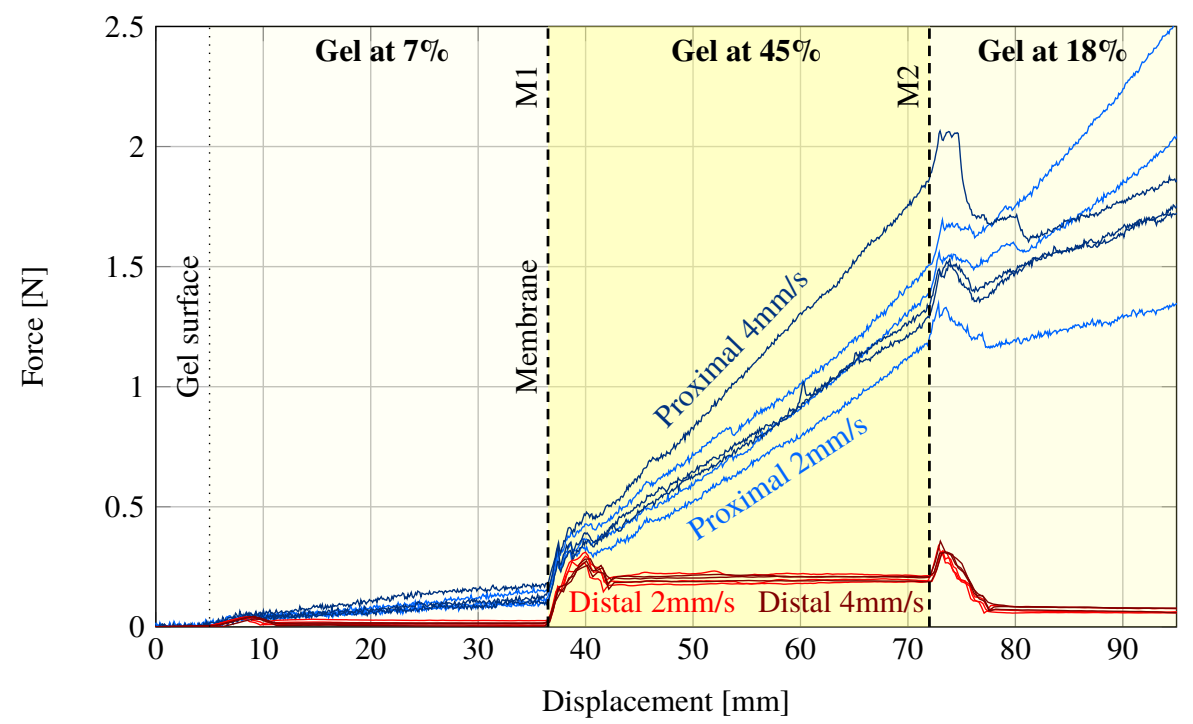

Fig. 4. Distal and proximal needle axial force measurement evolution with respect to the insertion depth at speed $2 \mathrm{~mm} / \mathrm{s}$ and $4 \mathrm{~mm} / \mathrm{s}$ in three various gels separated by membranes.

\section{Discussion}

With the considered instrumented needle, the ability to measure the force at the tip of the needle is proven to be clearly an added-value to build force feedback during needle insertion, as also observed in [7]. In fact, the insertion profile of the force measurement at the distal end of the needle allows us to detect in a simple way the puncture during the whole insertion. The phantom was here based on homogeneous layers. It will be interesting to further investigate force measurements with this design of needle based on Fabry-Perot interferometry, and how to make the signal processing robust to signals collected in in vivo conditions.

The approach we propose to reflect to the user events related to the needle progression is quite simple. We actually limit the number of information sent to the user, providing only one piece of information related to the nature of tissue and another one related to the occurrence of membrane puncture. As one can see, the absence of transition detection occurs equivalently for both transition (T12 and T23) and are not correlated with the absence or presence of a membrane (M1 or M2). The evaluation results are excellent (98\%) for the puncture detection which is very encouraging in terms of safety improvement during teleoperated needle insertion as the tactile feedback comes as a complementary information to the visual feedback provided by the medical imaging device. Puncture information to take a decision about needle progression could be sufficient, as they have a priori knowledge about the anatomy. However, the feedback about change of tissue seems also feasible, and it could be a valuable complementary source of information if unexpected changes occur. 


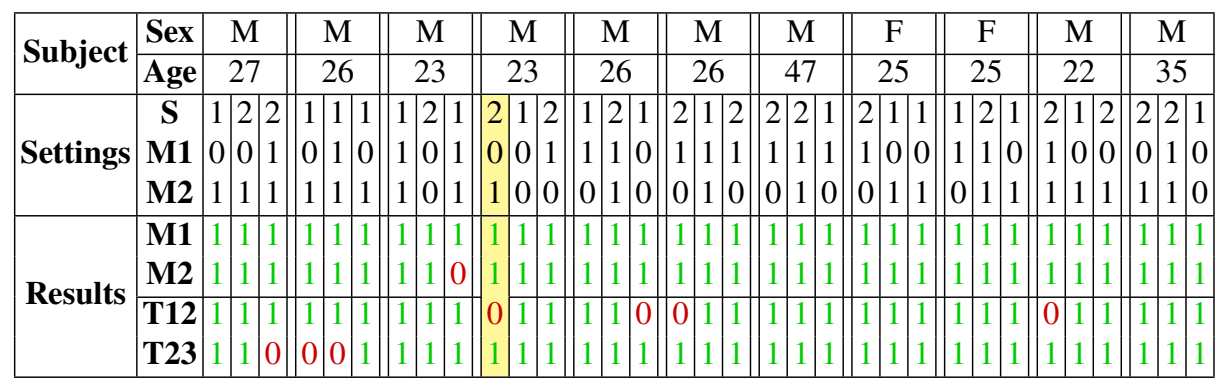

Table 1. Results of the experimental evaluation. The highlighted column can be interpreted as follows. The male subject is 23 years old. The first insertion has been set to an insertion speed of $4 \mathrm{~mm} / \mathrm{s}$ (speed \#2), simulating an absence of membrane M1 between the first and second gel layers, by not sending a signal to the tactuator, but with a membrane M2 between the second and third gel layers. The results are split in two parts: membrane puncture detection, and change of tissue. In case of the non-occurrence of membrane M1 puncture, the subject did not press the push button, which was correct (1). In case of membrane puncture, the ON/OFF pattern has been sent to the tactuator and the subject pressed the push button which was also correct (1). The second part of the evaluation is the ability to detect a change of tissue which is interpreted by an increase or decrease of the frequency of the moving line pattern. For the change between the first and second layers (T12 with no membrane M1) the subject did not detect any change (0). However, for the second change between the second and third layers (T23 with membrane M2), the subject did detect the change (1).

\section{Conclusion}

In this paper, the interest of needle tip force sensing capabilities in remote needle insertion was presented and discussed as well as its experimental evaluation in combination with tactile force feedback. The interest of needle tip force sensing is demonstrated by comparing the insertion profiles of distal versus proximal force sensing when inserted in a phantom. Based on the profile of tip force measurement, puncture and changes of tissues could be well established and reconstructed as a tactile information with two distinct dynamic patterns. The experimental evaluation on 11 subjects with a phantom demonstrates that it is possible to detect puncture event which could improve the safety during remote needle insertion. Other information as the change of tissues which seems understandable through a tactile feedback could be a crucial information to avoid critical zones.

\section{Acknowledgements}

This work was supported by the INTERREG Upper Rhine program from the ERDF (European Regional Development Fund), SPIRITS project (interreg-spirits.eu), and also by Investissements d'Avenir program (Robotex ANR-10-EQPX-44, Labex CAMI ANR-11- LABX-0004). The authors thank Sensoptic SA for the work on needle with tip force sensing capabilities and Help Tech $\mathrm{GmbH}$ for providing the Braille display. 


\section{References}

1. H. Schneegans, L. Rubbert, J. Rivera, S. Fifanski, P. Renaud, S. Henein, C. Baur. Fiber Optic Fabry-Perot Interferometry for a Biopsy Needle with Tip Force Sensing, IEEE IROS, Macau, China, 4-8 november 2019.

2. A. Pfeil, L. Barbé, B. Wach, R. Cazzato, A. Gangi, P. Renaud. Observations And Experiments For The Definition Of A New Robotic Device Dedicated To CT, CBCT And MRI-Guided Percutaneous Procedures. In proceedings of International Conference of the IEEE Engineering in Medicine and Biology Society. 2018.

3. L. Barbé, B. Bayle, E. Laroche, M. de Mathelin. User Adapted Control of Force Feedback Teleoperators: Evaluation and Robustness Analysis, in IEEE/RSJ International Conference on Intelligent Robots and Systems (IROS 2008), Nice, France, septembre 2008.

4. S. Elayaperumal, J. H. Bae, B. L. Daniel, and M. R. Cutkosky, Detection of membrane puncture with haptic feedback using a tip-force sensing needle. IEEE International Conference on Intelligent Robots and Systems, (Iros):3975-3981. 2014

5. R. Zhu , L. Rubbert, P. Renaud, U. Mescheder Determination of a tactile feedback strategy for use in robotized percutaneous procedures, 41st Annual International Conference of the IEEE Engineering in Medicine and Biology Society (EMBC), Berlin, Germany, IEEE, july 2019.

6. K. W. Ng, J. Q. Goh, S. L. Foo,P. H. Ting, T. K. Lee. Needle Insertion Forces Studies for Optimal Surgical Modeling. International Journal of Bioscience, Biochemistry and Bioinformatics. Vol. 3, 187-191 2013

7. S. Elayaperumal, J. H.Bae, D. Christensen, M. R. Cutkosky, B. L. Daniel, J. M. Costa, R. J. Black, F. Faridian, B. Moslehi. MR-compatible biopsy needle with enhanced tip force sensing, Joint Eurohaptics Conf. Symp. Haptic Interfaces Virtual Environ Teleoper Syst. 2015. 